The Astronomical JournaL, 117:238-246, 1999 January

(C) 1999. The American Astronomical Society. All rights reserved. Printed in U.S.A.

\title{
A REVISED AND EXTENDED CATALOG OF MAGELLANIC SYSTEM CLUSTERS, ASSOCIATIONS, AND EMISSION NEBULAE. II. THE LARGE MAGELLANIC CLOUD
}

\author{
Eduardo L. D. Bica, ${ }^{1,2,3}$ Henrique R. Schmitt,${ }^{1,2,3}$ Carlos M. Dutra, ${ }^{1,2,3}$ and Humberto L. Oliveira ${ }^{1,2,3}$ \\ Received 1998 July 9; accepted 1998 August 25
}

\begin{abstract}
A survey of extended objects in the Large Magellanic Cloud was carried out on the ESO/SERC R and J Sky Survey Atlases, checking entries in previous catalogs and searching for new objects. The census provided 6659 objects including star clusters, emission-free associations, and objects related to emission nebulae. Each of these classes contains three subclasses with intermediate properties, which are used to infer total populations. The survey includes cross identifications among catalogs, and we present 3246 new objects. We provide accurate positions, classification, and homogeneous measurements of sizes and position angles, as well as information on cluster pairs and hierarchical relation for superimposed objects. This unification and enlargement of catalogs is important for future searches of fainter and smaller new objects. We discuss the angular and size distributions of the objects of the different classes. The angular distributions show two off-centered systems with different inclinations, suggesting that the LMC disk is warped. The present catalog together with its previous counterpart for the SMC and the inter-Cloud region provide a total population of 7847 extended objects in the Magellanic System. The angular distribution of the ensemble reveals important clues on the interaction between the LMC and SMC.

Key words: catalogs — galaxies: ISM — galaxies: star clusters — galaxies: stellar content Magellanic Clouds
\end{abstract}

\section{INTRODUCTION}

The systematic study of the Large Magellanic Cloud (LMC) properties is nowadays possible partly thanks to cataloging efforts carried out throughout decades. Hodge (1975) reviewed the history of star cluster catalogs in the LMC, providing a census of 1614 objects at that time, considering overlapping entries. Hodge's (1960) catalog of red clusters, Shapley \& Lindsay's (1963) overall catalog, and Lyngå \& Westerlund's (1963) catalog of clusters in the outer parts of the LMC play a fundamental role in all aspects of research concerning these objects. Hodge \& Sexton (1966) provided an additional wide-angle general catalog for fainter clusters.

Hodge's (1980) deep plate survey sampled different LMC regions. He predicted a total cluster population of $\approx 5100$ objects, by considering completeness effects. Hodge (1988) studied additional fields finding new clusters and, by taking into account a population of 2053 cataloged clusters and completeness effects, reevaluated the total population down to $\approx 4200$ clusters.

A technique similar to that used by Hodge (1988) was employed by Kontizas, Metaxa, \& Kontizas (1988) in the LMC bar detecting new faint clusters and studying their internal dynamical properties. Several other studies have contributed with new objects, and provided accurate positions for previously cataloged ones (Lauberts 1982; Olszewski et al. 1988; Kontizas et al. 1990).

Stellar associations in the LMC have been cataloged and described by Lucke \& Hodge (1970), which has been a key reference for subsequent studies. In a recent study of such

\footnotetext{
${ }^{1}$ Departamento de Astronomia, IF-UFRGS, C.P. 15051, 91501-970 Porto Alegre, RS, Brazil.

${ }^{2}$ CNPq Fellow.

${ }^{3}$ bica@if.ufrgs.br, schmitt@if.ufrgs.br, dutra@if.ufrgs.br, humberto@ if.ufrgs.br.
}

objects Kontizas et al. (1994) found new associations in the LMC main body. Also, automated techniques have detected clusters in the northeast region of the LMC (Bhatia \& MacGillivray 1989), and associations in the interCloud region (Battinelli \& Demers 1992).

The fundamental catalogs of emission nebulae in the LMC are Henize's (1956), based on objective-prism and direct-exposure plates, and that by Davies, Elliot, \& Meaburn (1976), based on deeper $\mathrm{H} \alpha$ direct plates. Henize (1956) included planetary nebulae and stellar objects that are beyond the scope of the present study. Otherwise, both catalogs are dominated by $\mathrm{H}$ II regions. Some objects turned out to be supernova remnants (SNRs), which amounted to 32 in the LMC according to Mathewson et al. (1985). Two new supernova remnants have been recently detected by Smith et al. (1994).

This paper presents a unified deep catalog of star clusters, stellar associations, and emission nebulae in the LMC. It is a continuation of the work started by Bica \& Schmitt (1995, hereafter Paper I), which was devoted to the SMC and the inter-Cloud region (the stellar and $\mathrm{H}$ I bridge linking the Clouds; Irwin, Demers, \& Kunkel 1990; Grondin, Demers, $\&$ Kunkel 1992). Homogeneous catalogs form a database useful for systematic studies of the structure and dynamics of the Clouds (see Westerlund 1990 for a review), and for dating their objects in view of recovering their star formation history (see, e.g., Hodge 1973).

The paper is organized in the following way. In $\S 2$ we present the procedures employed in the construction of the catalog and show the results, giving examples of finding charts that can be built with the present catalog. In $\S 3$ we discuss the angular distribution of the different object types in the LMC, their relation to the $\mathrm{H}$ I distribution, and also study their size distribution. In $\S 4$ we use the present LMC catalog together with that of the SMC and Bridge regions (Paper I) to infer possible effects of the LMC and SMC interaction. The conclusions of this work are given in $\S 5$. 


\section{LMC CATALOG}

In the 70s the cataloged objects were mostly contained in the LMC Atlas (Hodge \& Wright 1967). In Table 1 we chronologically gathered references before and after the LMC Atlas, indicate their corresponding acronyms, number of entries and the object types that they basically include (C, A, or $\mathrm{N}$ for star cluster, association and emission nebula, respectively). Discoveries of new objects have been numerous, but also new designations for previous objects have been created and cross-checking them was necessary.

The present revision also includes the LMC objects in the NGC and IC catalogs, and the objects in the ESO catalog (Lauberts 1982). Some catalogs of bright stars and/or of emission line stars include some compact star clusters (Heydari-Malayeri, Magain, \& Remy 1988, 1989; HeydariMalayeri \& Hutsemékers 1991; Heydari-Malayeri et al. 1993), and compact H II regions (e.g., Heydari-Malayeri \& Testor 1983). Their acronyms were used in the present study in order not to create new designations: HD or HDE, LMC-S-Henize (1956); Sk-Sanduleak (1969).

The objects were examined and measured on the ESO/ SERC R and J Sky Survey Schmidt films at the Instituto de Física, UFRGS. The measurements for calculating accurate coordinates relative to reference stars, as well as object sizes and position angles, were carried out with a $7 \times$ magnifying lens equipped with a graduated glass providing a length resolution of $0.05 \mathrm{~mm}$ (which converts to less than 4 " on the ESO Schmidt films) and a precision in position angles of $\pm 5^{\circ}$.

The procedures employed to built the LMC catalog follow those explained in detail in Paper I, so we briefly recall them in the following: (i) digitalization of the original coordinates and precession to the epoch 1950 when necessary; (ii) identification of the objects on the Schmidt films using all the available information in terms of coordinates, descriptions, identification plates and finding charts in the original papers and in the LMC Atlas (Hodge \& Wright 1967); (iii) equivalent objects cross-identified in different catalogs were merged into a single catalog line; (iv) transparent overlay charts were generated via computer to the same scale as the ESO/SERC Schmidt films; (v) accurate coordinates, whenever available, replaced original ones, and for the rest, accurate positions were measured with respect to reference stars; (vi) major and minor diameters, as well as position angle of the major axis were measured.

We illustrate in Table 2 the first five lines of the catalog; the whole catalog is available through the electronic version by link to the University of Chicago Press archive. The table is organized in the following way. Column (1) gives the Sky Survey plate and quadrant where the object is best

TABLE 1

\begin{tabular}{|c|c|c|c|}
\hline Acronym & Reference & Entries & Types \\
\hline LMC-N ........... & Henize 1956 & 407 & $\mathrm{~N}$ \\
\hline $\mathrm{H} \ldots \ldots \ldots \ldots \ldots \ldots$ & Hodge 1960 & 12 & $\mathrm{C}$ \\
\hline SL $\ldots \ldots \ldots \ldots \ldots \ldots$ & Shapley \& Lindsay 1963 & 898 & $\mathrm{C}$ \\
\hline LW $\ldots \ldots \ldots \ldots \ldots$ & Lyngå \& Westerlund 1963 & 435 & $\mathrm{C}$ \\
\hline HS .............. & Hodge \& Sexton 1966 & 457 & $\mathrm{C}$ \\
\hline $\mathrm{U}^{\mathrm{a}} \ldots \ldots \ldots \ldots \ldots \ldots$ & Hodge \& Sexton 1966 & 6 & $\mathrm{C}$ \\
\hline LH ................. & Lucke \& Hodge 1970 & 122 & $\mathrm{~A}$ \\
\hline Rob ............... & Robertson 1974 & 1 & $\mathrm{C}$ \\
\hline LMC-DEM ........ & Davies et al. 1976 & 352 & $\mathrm{~N}$ \\
\hline $\mathrm{C} \ldots \ldots \ldots \ldots \ldots \ldots$ & Hodge 1975 & 11 & $\mathrm{C}$ \\
\hline $\mathrm{H} 80-\mathrm{F} \ldots \ldots \ldots \ldots$ & Hodge 1980 & 132 & $\mathrm{C}$ \\
\hline SGshell-LMC ...... & Meaburn 1980 & 9 & $\mathrm{~N}, \mathrm{~A}$ \\
\hline Shapley- ............ & van den Bergh 1981 & 9 & $\mathrm{~A}, \mathrm{~N} \mathrm{C}$ \\
\hline SNR .............. & Mathewson et al. 1983 & 25 & $\mathrm{~N}$ \\
\hline HT $\ldots \ldots \ldots \ldots \ldots \ldots$ & Heydari-Malayeri \& Testor 1983 & 4 & $\mathrm{~N}$ \\
\hline $\mathrm{LT}-\ldots \ldots \ldots \ldots \ldots$ & Lortet \& Testor 1984 & 6 & $\mathrm{C}$ \\
\hline SNR $\ldots \ldots \ldots \ldots \ldots$ & Mathewson et al. 1984 & 2 & $\mathrm{~N}$ \\
\hline SNR $\ldots \ldots \ldots \ldots \ldots \ldots$ & Mathewson et al. 1985 & 7 & $\mathrm{~N}$ \\
\hline HNT ............... & Heydari-Malayeri et al. 1987 & 1 & $\mathrm{NC}$ \\
\hline M-OB ............. & Melnick 1987 & 6 & $\mathrm{C}, \mathrm{A}$ \\
\hline KMK88- .......... & Kontizas et al. 1988 & 93 & $\mathrm{C}$ \\
\hline $\mathrm{H} 88-\ldots \ldots \ldots \ldots \ldots$ & Hodge 1988 & 255 & $\mathrm{C}$ \\
\hline BH $\ldots \ldots \ldots \ldots \ldots \ldots$ & Bhatia \& Hatzidimitriou 1988 & 138 & $\mathrm{C}$ \\
\hline OHSC $\ldots \ldots \ldots \ldots \ldots$ & Olszewski et al. 1988 & 37 & $\mathrm{C}$ \\
\hline $\mathrm{BM} \ldots \ldots \ldots \ldots \ldots$ & Bhatia \& MacGillivray 1989 & 282 & A \\
\hline KMHK ........... & Kontizas et al. 1990 & 1762 & $\mathrm{C}$ \\
\hline IDK $\ldots \ldots \ldots \ldots \ldots$ & Irwin et al. 1990 & 1 & $\mathrm{~A}$ \\
\hline BRHT ............. & Bhatia et al. 1991 & 128 & $\mathrm{C}$ \\
\hline $\mathrm{BD} \ldots \ldots \ldots \ldots \ldots$ & Battinelli \& Demers 1992 & 5 & A \\
\hline $\mathrm{BCD} \ldots \ldots \ldots \ldots \ldots$ & Bica et al. 1992 & 1 & $\mathrm{C}$ \\
\hline SNR $\ldots \ldots \ldots \ldots \ldots$ & Smith et al. 1994 & 2 & $\mathrm{~N}$ \\
\hline KKDAB- .......... & Kontizas et al. 1994 & 7 & A \\
\hline BCDSP ............. & Bica et al. 1996 & 8 & $\mathrm{C}, \mathrm{A}$ \\
\hline ZHT ............. & Zaritsky et al. 1997 & 16 & $\mathrm{C}$ \\
\hline ZHT-SP & Zaritsky et al. 1997 & 10 & $\mathrm{C}$ \\
\hline SESG ............. & Santiago et al. 1998 & 2 & $\mathrm{C}$ \\
\hline BSDL ............. & This paper & 3246 & $\mathrm{C}, \mathrm{N}, \mathrm{A}$ \\
\hline
\end{tabular}

a Entries from a deep plate presented by Hodge \& Sexton 1966.

b Entries from a list of possible objects in Zaritsky et al. 1997. 
TABLE 2

Revised AND EXTENDED CATALOG

\begin{tabular}{|c|c|c|c|c|c|c|c|c|}
\hline $\begin{array}{l}\text { Plate } \\
\text { (1) }\end{array}$ & $\underset{(2)}{\text { Name }}$ & $\begin{array}{l}\text { R.A. (2000) } \\
\text { (3) }\end{array}$ & $\begin{array}{l}\text { Decl. (2000) } \\
\text { (4) }\end{array}$ & $\begin{array}{c}T \\
(5)\end{array}$ & $\begin{array}{c}D_{\max } \\
\text { (arcmin) } \\
\quad(6)\end{array}$ & $\begin{array}{c}D_{\min } \\
\text { (arcmin) } \\
\quad(7)\end{array}$ & $\begin{array}{l}\text { P.A. } \\
\text { (deg) } \\
\text { (8) }\end{array}$ & $\begin{array}{c}\text { Remarks } \\
(9)\end{array}$ \\
\hline $54 \mathrm{se} . . . \ldots$. & NGC 1466, SL 1, LW1 & 34433 & -714017 & $\mathrm{C}$ & 3.50 & 3.50 & ... & \& ESO 54-SC16, KMHK 1 \\
\hline $32 \mathrm{nw} . . . .$. & BD 74 & 35350 & -735518 & A & 4.20 & 2.10 & 10 & \\
\hline $32 \mathrm{nw} . . . .$. & BD 75 & 40203 & -740410 & A & 2.00 & 1.40 & 150 & \\
\hline $32 \mathrm{nw} . . . .$. & IDK 6, BD 76 & 41929 & -735349 & A & 7.00 & 6.00 & 10 & \\
\hline $32 \mathrm{nw} . . . .$. & BD 77 & 42111 & -734311 & A & 5.00 & 5.00 & $\ldots$ & \\
\hline
\end{tabular}

NoTE.-Table 2 is presented in its entirety in the electronic edition of the Astronomical Journal. A portion is shown here for guidance regarding its form and content. Units of right ascension are hours, minutes, and seconds, and units of declination are degrees, arcminutes, and arcseconds.

seen. Column (2) gives the object cross-identification in the different catalogs; we have considered as equivalent objects $\mathrm{H}$ II regions and their embedded stellar associations of comparable extent; some objects were separated in two or more parts and consequently the designation is complemented with " $\mathrm{n}$ " for northern part, "se" for southeastern part, etc. We present 3246 new objects, $\approx 49 \%$ of the unified catalog. In the SMC (Paper I) new objects amounted to $24 \%$ only,

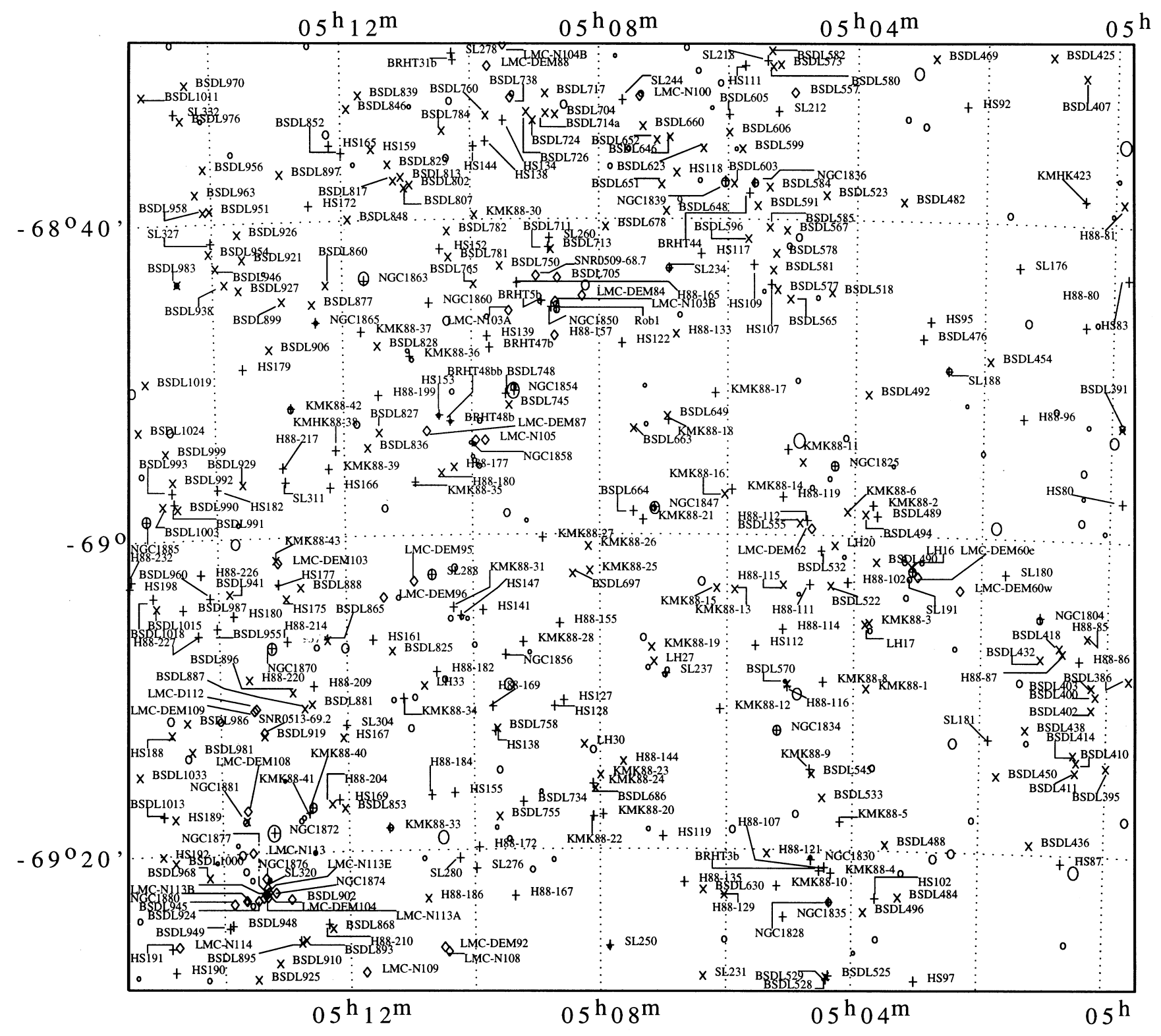

FIG. $1 a$

FIG. 1.-Examples of finding charts (J2000.0) that can be generated with the present catalog. $(a)$ and $(b)$ show high- and low-density regions, respectively. Plus signs identify clusters, crosses associations and diamonds nebulae. The figures also include reference stars from the Guide Star Catalog, identified by ellipses. The fainter stars shown are of magnitude 13 and 14 . 


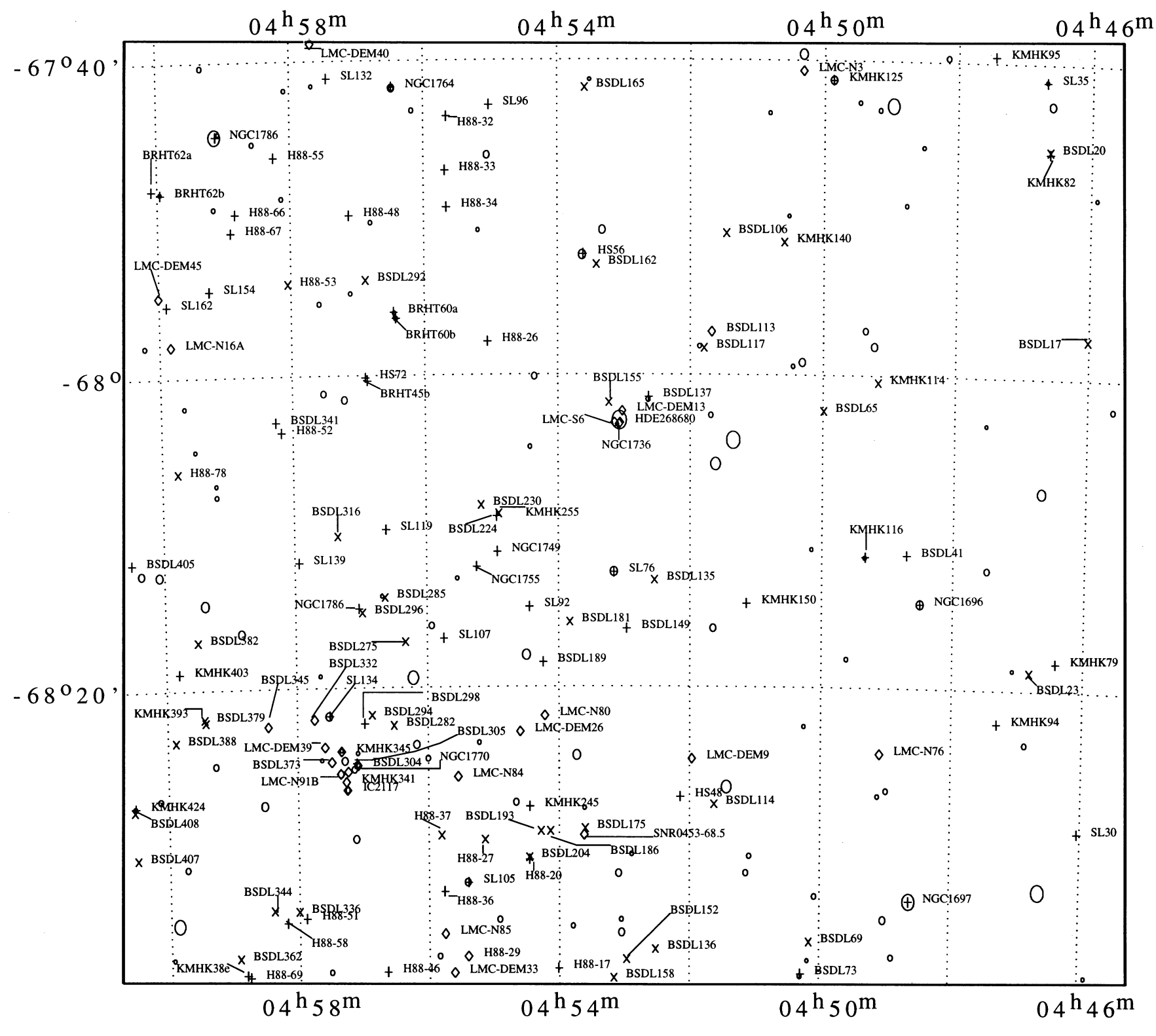

FIG. $1 b$

showing that previous surveys had explored more efficiently the SMC, possibly because of its smaller angular size. Columns (3) and (4) provide, respectively, right ascension and declination precessed to J2000.0. Column (5) indicates the object type following Paper I: the sequence C, CA, AC to $A$ is essentially one of density, where objects with the higher density of stars are classified as $\mathrm{C}$, and objects with the lower density as A. Objects NA and NC are stellar systems clearly related to emission, while $\mathrm{N}$, in this particular table, refers to known supernova remnants. AN and $\mathrm{CN}$ are stellar systems with traces of emission. Columns (6) and (7) give the size of the object's major and minor axes, respectively. Column (8) gives the position angle of the major axis $\left(0^{\circ}=\mathrm{N}, 90^{\circ}=\mathrm{E}\right)$. Finally, column $(9)$ gives remarks: " $\mathrm{mT}$ " and " $\mathrm{mP}$ " indicate member of triple or pair, respectively. A hierarchical indication is given for objects embedded in or superimposed on larger ones, where "in" suggests a possible physical connection, while "sup" suggests a projection. Also, "\&" indicates additional designation(s), which complement column (2) of the catalog.
We show in Figures $1 a$ and $1 b$ two finding charts that can be built with this catalog and IRAF task SKYMAP. Figure $1 a$ shows a dense region, centered in the LMC bar, while Figure $1 b$ shows a less crowded region, located to the West of the bar. These figures identify objects of different basic types (A, C, and N) by different symbols, label them, and also include field stars for reference. The stars were obtained from the Guide Star Catalog (Jenkner et al. 1990 and references therein). Notice that some star clusters are also entries in the Guide Star Catalog.

\subsection{Census}

We show in Table 3 the census of the subclasses of objects in the present work. The most numerous objects are clusters and associations. Including intermediate classifications, there are 2577 clusters $(\mathrm{C}+\mathrm{CA}+\mathrm{CN}), 2883$ emission-free associations $(\mathrm{A}+\mathrm{AC}+\mathrm{AN})$ and 1199 emission line related objects $(\mathrm{N}+\mathrm{NC}+\mathrm{NA})$. We basically confirm Hodge's (1988) prediction of $\approx 4200$ star clusters in the LMC, by adding to the $\mathrm{C}+\mathrm{CA}+\mathrm{CN}$ the subclasses $\mathrm{AC}$, consisting 
TABLE 3

\begin{tabular}{lr}
\multicolumn{2}{c}{ LMC Census } \\
\hline \hline Classification & Number \\
\hline C $\ldots \ldots \ldots \ldots \ldots$ & 1808 \\
CA $\ldots \ldots \ldots \ldots$. & 717 \\
CN $\ldots \ldots \ldots \ldots$. & 52 \\
A $\ldots \ldots \ldots \ldots \ldots$. & 1436 \\
AC $\ldots \ldots \ldots \ldots$. & 1300 \\
AN $\ldots \ldots \ldots \ldots$. & 147 \\
NC $\ldots \ldots \ldots \ldots$. & 212 \\
NA $\ldots \ldots \ldots \ldots$. & 952 \\
N $\ldots \ldots \ldots \ldots \ldots$ & 35 \\
\hline
\end{tabular}

of somewhat looser objects, and NC, which are newly formed star clusters, totaling 4089 objects.

Notice that the definition of association in the present study (and also in Paper I) is related to the density of stellar systems, so they are not necessarily young as the traditional OB associations, but $\mathrm{OB}$ associations (Lucke \& Hodge 1970) are included in this class as A, NA, or AN. The densities of stars in the lowest density objects as counted on Sky Survey plates are typically 5-10 stars per square minute, a factor $\approx 2$ larger than in surrounding regions. They often have irregular or elongated shapes. Detection efficiencies certainly depend on background star densities, and should become critical in the bar region. In crowded regions, younger objects (higher surface brightness) are expected to be more easily detected than older ones.

As to the emission related objects, apart from the 35 known supernova remnants (notice that LMC-DEM316 was separated into two components), the remaining 1164 objects are mostly $\mathrm{H}$ II regions, with a wide range in dimensions $(\S 3.2)$.

\section{3. $\mathrm{LMC}$}

\subsection{Angular Distribution}

Figures $2 a, 2 b, 2 c$, and $2 d$ show the angular distribution of: (a) all extended objects; (b) clusters; (c) associations; (d) emission line objects. Superimposed on Figures $2 b, 2 c$, and $2 d$ is the $\mathrm{H}$ I surface density distribution in the LMC (Mathewson \& Ford 1984). Figure $2 a$ clearly shows two stellar systems, a nearly circular dense internal disk with dimensions $\approx 6^{\circ} \times 7^{\circ}$, and a less dense outer disk with $\approx 10^{\circ} \times 14^{\circ}$. The centers of the two systems are separated by approximately $1^{\circ}$. Assuming that the intrinsic distributions are circular, the axial ratios imply inclinations of $\approx 45^{\circ}$ and $\approx 30^{\circ}$ for the outer and inner systems, respectively. Such tilts would imply that the LMC disk is warped. The line of nodes of the outer system is approximately aligned with the N-S direction, while that of the inner system is aligned along P.A. $\approx 130^{\circ}$. The existence of two disk systems (Fig. 2a), can explain why estimates of geometrical and kinematical parameters depend on the type of object used to determine them (Westerlund 1990 and references therein).

In the southern outer region of the LMC there occurs a structure in the object distribution (Fig. 2, see also Fig. 4a), already described by Lyngå \& Westerlund (1963), and possibly related to a spiral arm (de Vaucouleurs 1955). The increased number of objects cataloged in the present study shows this structure in more detail. In order to test its significance we counted objects in $1^{\circ} \times 1^{\circ}$ boxes along the disk edge and slightly more internal control zones. The box centers, position angles, angular distances from the LMC bar center and counts are given in Table 4. Considering $N^{1 / 2}$ fluctuations for the count $N$, it can be concluded that the southern structure is statistically significant from position angles $\approx 140^{\circ}$ to $\approx 220^{\circ}$. Counts of field stars might reveal whether this is an arm, a partial ring or part of a complete ring, which could be blurred by contamination in the eastern and northern regions and hidden by the offcentered high-density inner disk to the west. Irwin (1991) presented LMC isopleths for field stars from machine-based measurements of UKST plates. He used horizontal branch/ giant clump stars, thus eliminating younger disk components. The southern structure is clear, and an equally dense region is seen all around the LMC, which could suggest the presence of a ring, but unfortunately in his Figure 3 adjacent control zones are available only for the southern part.

The angular distribution of star clusters (Fig. 2b) clearly shows the LMC bar in the central region, which is not the case in the angular distribution of associations (Fig. 2c). This is due to the fact that the stellar population of the bar has an important intermediate age component (Hardy et al. 1984; Bica, Clariá, \& Dottori 1992), and that star formation in the last $3 \times 10^{7} \mathrm{yr}$ has been important in the NE of the bar (Dottori et al. 1996), consequently enhancing the number of $\mathrm{OB}$ associations in that region. In Figure $2 b$, the $\mathrm{H}$ I distribution shares a similar center with the inner disk system and the $10 \times 10^{19}$ atoms $\mathrm{cm}^{-2}$ contour does not encompass the outer cluster distribution in the north east area. Such asymmetries between the outer stellar disk and the $\mathrm{H}$ I distribution, again point to the perturbed nature of the LMC.

In Figure $2 c$ there is a strong concentration of associations in a region depleted of $\mathrm{H}$ I at R.A. $\approx 5^{\mathrm{h}} 32^{\mathrm{m}}$ and $\delta \approx-66^{\circ} 49^{\prime}$. This region corresponds to Shapley-III, where $\mathrm{H}$ I has probably been consumed and dispersed by winds arising from intense star formation at approximately $10^{7} \mathrm{yr}$.

The ongoing star formation is seen in the distribution of emission line objects (Fig. $2 d$ ), which is dominated by $\mathrm{H}$ II regions (some supernova remnants are also present). The necessary $\mathrm{H}$ I surface density for star formation is between 40 and $100 \times 10^{19}$ atoms $\mathrm{cm}^{-2}$, similar to the value found

TABLE 4

COUNTS IN THE LMC OUTER PARTS

\begin{tabular}{crrccl}
\hline \hline & & & P.A. & $r$ & \\
R.A. (2000) & Decl. (2000) & $N \pm \sqrt{N}$ & $\begin{array}{c}r \\
(\mathrm{deg})\end{array}$ & $\begin{array}{c}\text { (deg) } \\
\text { Zone }\end{array}$ \\
\hline 044934 & -723526 & $21 \pm 4.6$ & 215 & 4.0 & Struc. \\
044939 & -734732 & $12 \pm 3.5$ & 206 & 5.0 & Struc. \\
045844 & -750357 & $23 \pm 4.8$ & 194 & 6.0 & Struc. \\
052321 & -750601 & $11 \pm 3.3$ & 178 & 5.8 & Struc. \\
054545 & -750135 & $10 \pm 3.2$ & 164 & 6.0 & Struc. \\
060424 & -740043 & $9 \pm 3.0$ & 148 & 5.6 & Struc. \\
061328 & -723328 & $8 \pm 2.8$ & 130 & 5.1 & Struc. \\
061951 & -711455 & $12 \pm 3.5$ & 112 & 5.1 & Struc. \\
061540 & -700054 & $11 \pm 3.3$ & 99 & 4.7 & Struc. \\
051055 & -734819 & $3 \pm 1.7$ & 191 & 4.6 & Control \\
052914 & -735500 & $5 \pm 2.2$ & 173 & 4.7 & Control \\
055101 & -734454 & $3 \pm 1.7$ & 155 & 4.9 & Control \\
055444 & -722938 & $6 \pm 2.4$ & 125 & 4.0 & Control \\
060227 & -710302 & $10 \pm 3.2$ & 118 & 4.8 & Control \\
060229 & -695728 & $9 \pm 3.0$ & 101 & 3.6 & Control \\
\hline
\end{tabular}

NoTE.-Units of right ascension are hours, minutes, and seconds, and units of declination are degrees, arcminutes, and arcseconds. 

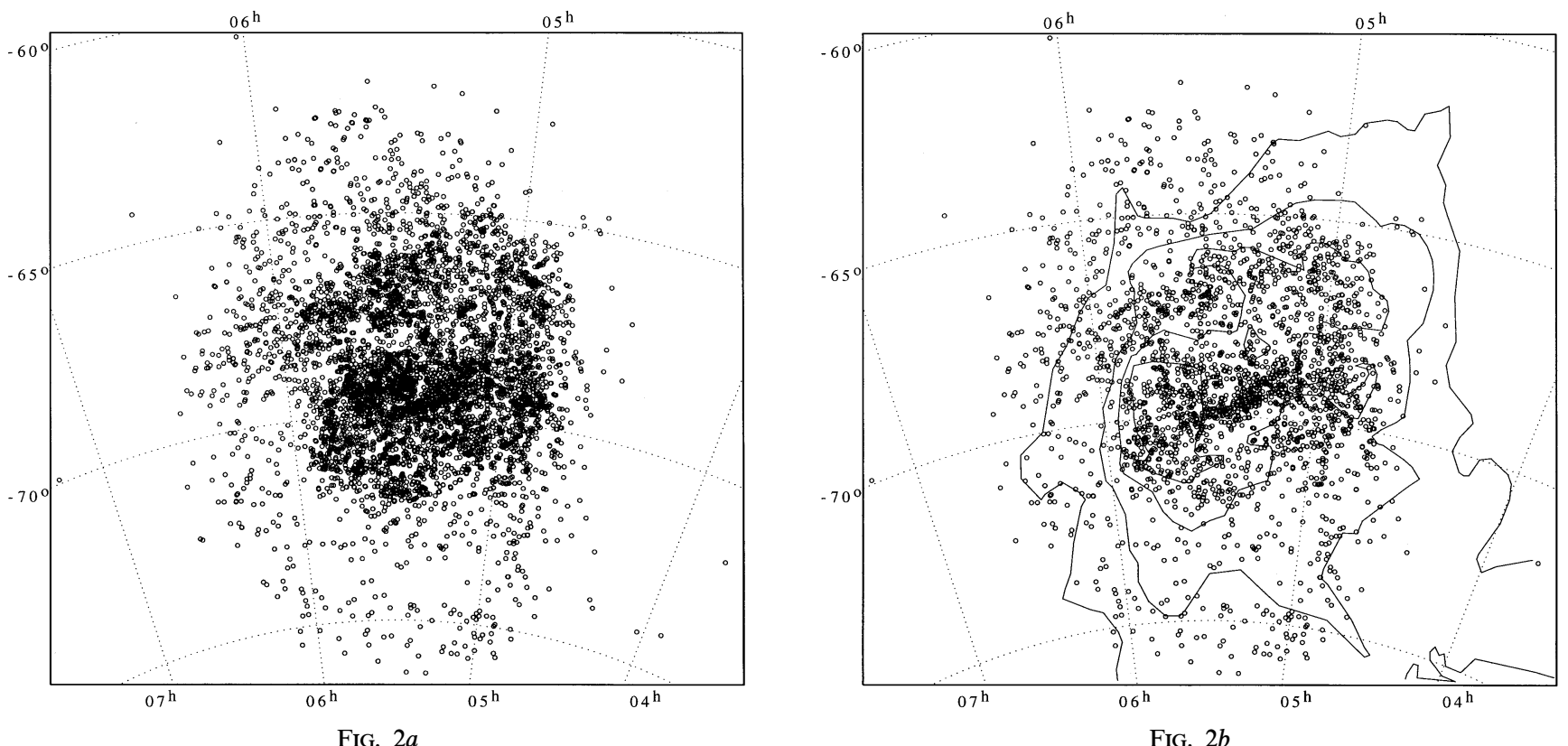

FIG. $2 b$
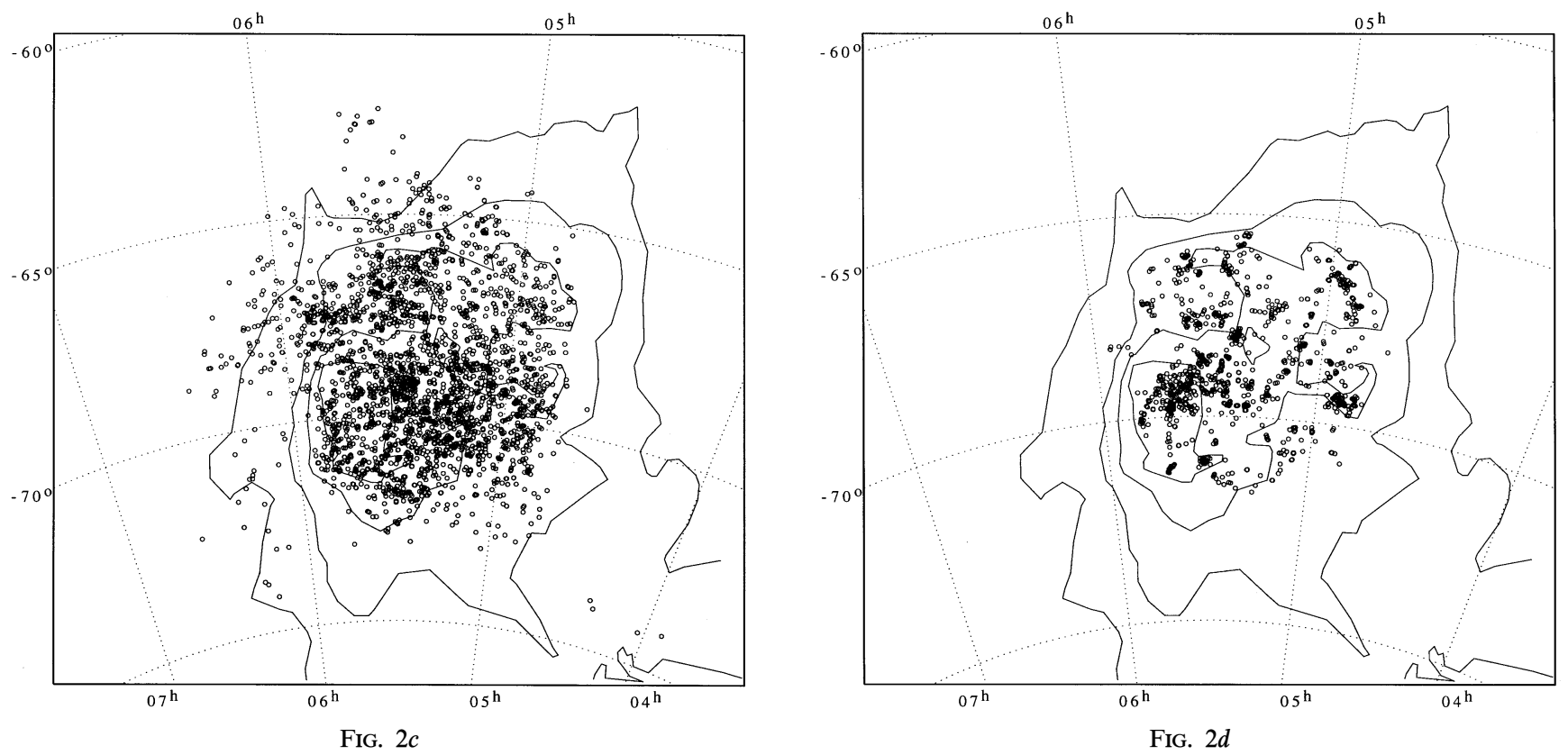

FIG. 2.-LMC angular distribution of $(a)$ all objects; $(b)$ star clusters; $(c)$ emission-free associations; $(d)$ objects with emission. H I surface density contours from Mathewson \& Ford (1984) for 10, 40, 100 and $200 \times 10^{19}$ atoms $\mathrm{cm}^{-2}$ are shown, increasing for outside to inside.

for the SMC in Paper I. The densest H I distribution occurs to the south of the 30 Doradus complex.

Since our classification from clusters to associations is one of density, some very loose objects are not necessarily young. The distribution of the loose objects (Fig. 2c) contains some quite far from the center of the LMC. They could be older than typical associations, corresponding to systems in the process of evaporation. Indeed, Wielen (1971) found evidence that, in the Galaxy, the dissolution of open clusters is an important phenomenon. One of the possibilities to explain the gap of clusters in the range 4-9 Gyr in the LMC (Da Costa 1991), is their dissolution (Geisler et al. 1997). This effect is expected in a wide range of ages. For example, Wielen (1988) estimated that a typical dissolution time is $\approx 2 \mathrm{Gyr}$ for a cluster in the outer LMC with an initial mass of $500 M_{\odot}$ and a core radius of $1 \mathrm{pc}$. It would be important to check by means of color magnitude diagrams and luminosity functions, the age and dynamical state of these outlying loose stellar systems. Some loose stellar systems occur in the vicinity or are pairs with dense star clusters (Table 2). $\mathrm{N}$-body simulations of star clusters with differing masses can produce as outcome loose dissolving systems from the less massive component, depending on the collision conditions (Oliveira, Dottori, \& Bica 1998). The generalized present definition of loose systems may encompass objects originated by this mechanism. Loose stellar systems are key objects to understand timescales and mechanisms by which star clusters and associations feed the field population.

\subsection{Size Distribution}

We show in Figures $3 a, 3 b$, and $3 c$ the distributions of sizes (mean of major and minor axes in Table 2), respec- 


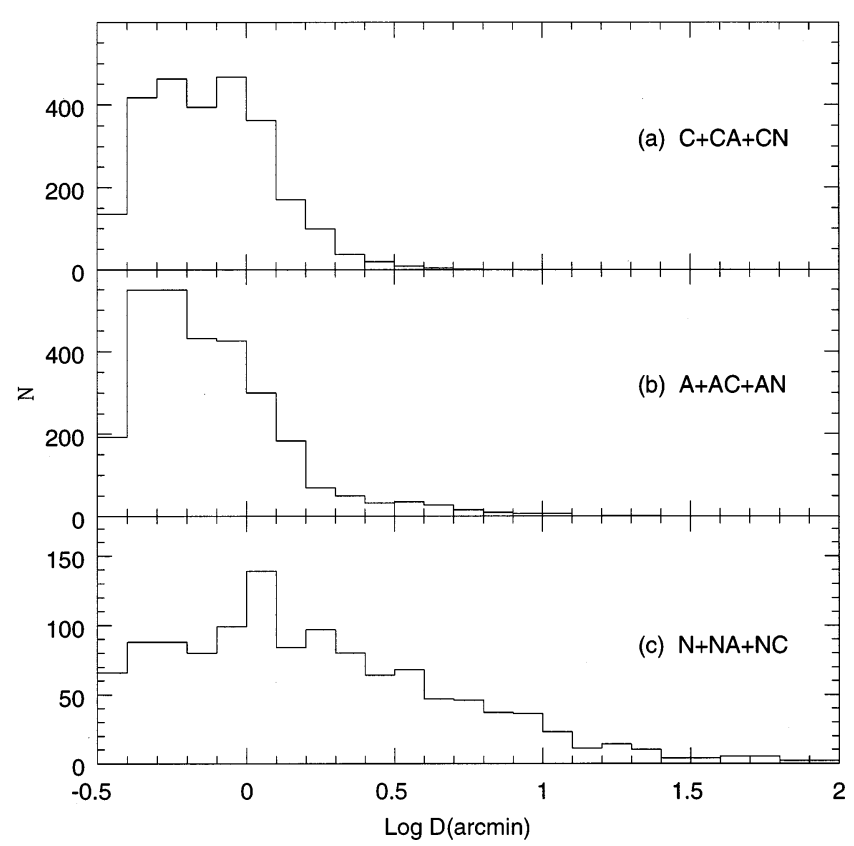

FIG. 3.-LMC size distributions of: (a) star clusters $(\mathrm{C}+\mathrm{CN}+\mathrm{CA})$; (b) emission-free associations $(\mathrm{A}+\mathrm{AC}+\mathrm{AN})$; (c) objects with emission $(\mathrm{N}+\mathrm{NA}+\mathrm{NC})$.

tively, for the star clusters $(\mathrm{C}+\mathrm{CN}+\mathrm{CA})$, the associations $(\mathrm{A}+\mathrm{AN}+\mathrm{AC})$ and the emission nebulae $(\mathrm{NA}+\mathrm{NC}+\mathrm{N})$. The largest star clusters have log $D\left(^{\prime}\right) \sim 0.7\left(D=5^{\prime}\right)$ and the number increases strongly toward smaller sizes down to $\log D\left({ }^{\prime}\right) \sim 0.0\left(D=1^{\prime}\right)$. Subsequently, there occurs a plateau and finally a drop for log $D\left(^{\prime}\right)<-0.4\left(D \sim 24^{\prime \prime}\right)$, where the number of objects in the bins is strongly affected by the detection limit.

The size distribution of the associations (Fig. $3 b$ ) shows a behavior similar to that of the star clusters, but having some objects considerably larger, with $\log D\left(^{\prime}\right)>1\left(D>10^{\prime}\right)$. In the case of the SMC and Bridge (Paper I), there is a sharp drop in the distribution of sizes of associations at log $D\left({ }^{\prime}\right) \approx 0.7$, which is due to the objects located in the Bridge. There is not such a drop in the LMC histogram. Notice the large population of small associations $\left[\log D\left({ }^{\prime}\right) \sim-0.2\right]$. Many of these loose objects are present in previous LMC cluster catalogs, as was the case also for the SMC (Paper I).

The distribution of objects containing emission (Fig. 3c) presents a flatter distribution, which attains very large values, some of which larger than $1^{\circ}(\approx 1 \mathrm{kpc})$. We point out that the latter structures correspond to supergiant shells (Meaburn 1980) and Shapley constellations (van den Bergh 1981), as well as large structures such as LMC-N135 (Henize 1956). Among the smallest objects there occur compact $H$ II regions with $D \leq 1^{\prime}$ (see, e.g., HeydariMalayeri \& Testor 1983).

\section{MAGELLANIC SYSTEM ANGULAR DISTRIBUTION}

The LMC catalog in conjunction with that of the SMC and Bridge regions (Paper I), can be used to study the overall angular distribution of extended objects in the Magellanic System, and their relation to the H I distribution.

The total number of cataloged extended objects in the Magellanic System amounts to 7847 entries: 3131 classified as clusters $(\mathrm{C}+\mathrm{CA}+\mathrm{CN}), 3226$ associations essentially free of emission ( $\mathrm{A}+\mathrm{AC}+\mathrm{AN})$, and 1490 objects related to emission $(\mathrm{N}+\mathrm{NA}+\mathrm{NC})$. Figure $4 a$ presents the angular distribution of all objects, where we can clearly see the interacting nature of the LMC and SMC, shown by the bridge of objects between the two galaxies, as well as by the distortions occurring in the main bodies and beyond.

We show in Figure $4 b$ the angular distribution of star clusters, with the Matthews \& Ford (1984) H I contours. Notice that clusters do not trace the $\mathrm{H}$ I bridge, extending only as far as the SMC Wing. As pointed out in $\S 3.1$, the outer $\mathrm{H}$ I contour $\left(10 \times 10^{19}\right.$ atoms $\left.\mathrm{cm}^{-2}\right)$ does not encompass the NE part of the LMC cluster distribution, but encompasses essentially all the SMC clusters. The low $\mathrm{H}$ I contours are not symmetrically distributed with respect to the cluster centroids, again denoting a perturbation in the $\mathrm{H} \mathrm{I}$ and stellar disks.

Figure $4 c$ shows the distribution of associations, which follow the $\mathrm{H}$ I distributions better than the star clusters, indicating that the nature of the loose objects is mostly young. In particular, they populate the $\mathrm{H}$ I bridge region. These objects are also abundant in the SMC wing and in the main body of both galaxies. Finally, Figure $4 d$ presents the distribution of objects related to emission, which are mostly contained inside higher H I contours $(\S 3.1$ and Paper I). In the case of the LMC we can see some structures, in particular the 30 Doradus complex and concentrations related to Shapley constellations. As pointed out in $\S 3.1$, the LMC distribution of young objects appears to be nearly face-on, while the distribution in the SMC is more concentrated along an axis, which might be interpreted as a nearly edge-on disk. The $200 \times 10^{19}$ atoms cm ${ }^{-2} \mathrm{H}$ I contour in the SMC is as well very elongated, also resembling an edge-on disk, with a bump in the direction of the Wing, which appears to be present also in the SMC nebulae distribution.

The above figures show features possibly arising from the LMC and the SMC interaction, whose basic characteristics can be inferred from available $N$-body simulations of a disk galaxy and a less massive ( $\approx$ a factor 5 ) compact companion. Hernquist \& Weil (1993) computed an axial collision, while Quinn, Hernquist, \& Fullagar (1993) computed a collision for a circular orbit inclined $30^{\circ}$ relative to the primary galaxy disk plane producing, respectively, a prominent outer ring, and a warping in the disk component. Both simulations produce disk thickening, and create high and low surface brightness components. Color-magnitude diagrams showed evidence of depth effects in NE region of the LMC (Bica et al. 1998), and the present LMC results suggest a warping between a high and low surface brightness disk components, together with a significant outer structure in the southern part ( 33.1$)$. This simple comparison suggests that an inclined nearly axial collision might reproduce the basic structures seen in the LMC disk. A detailed model should include gas-rich components for both Clouds, and the dominant Milky Way potential. It should also describe the gas and young stellar bridge between the Clouds (Irwin et al. 1990; Grondin et al. 1992), and the distortions in the SMC (Paper I).

\section{CONCLUDING REMARKS}

We have unified the catalogs of star clusters, associations and emission nebulae in the LMC. In the case of this revision we have detected 3246 new objects, totaling 6659 entries in the general catalog. We provide cross identifica- 


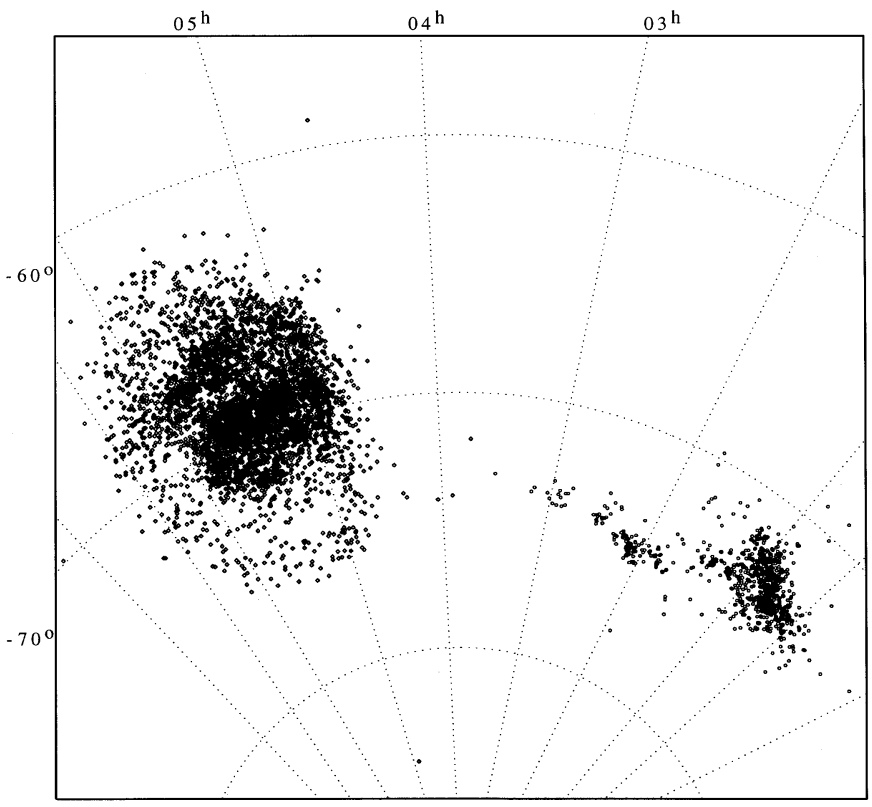

FIG. $4 a$

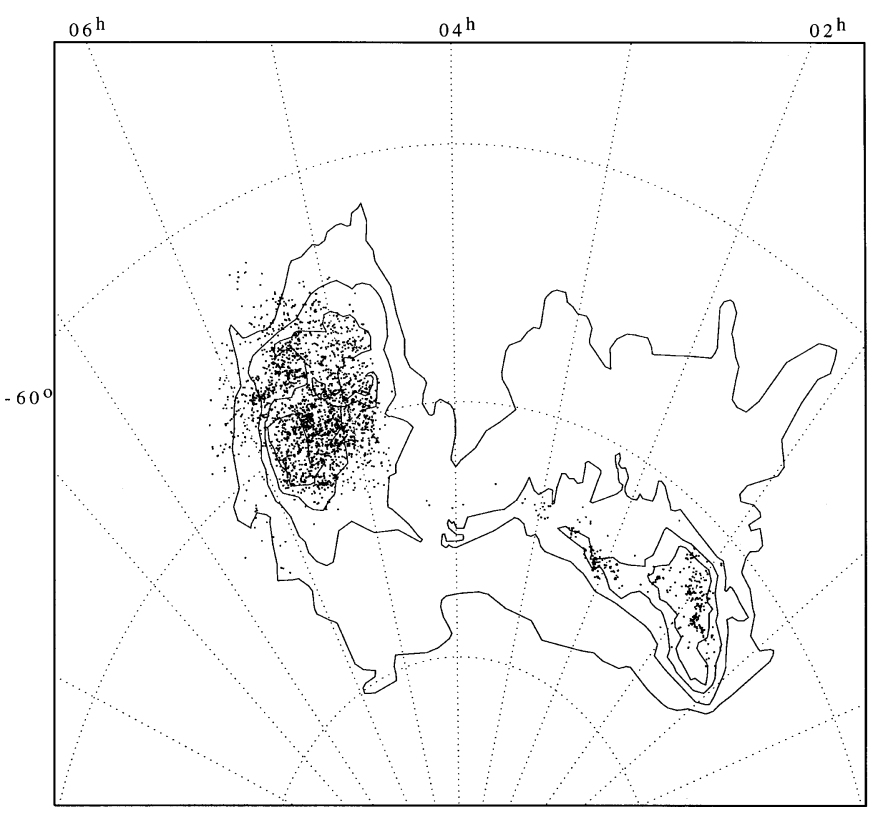

FIG. $4 c$

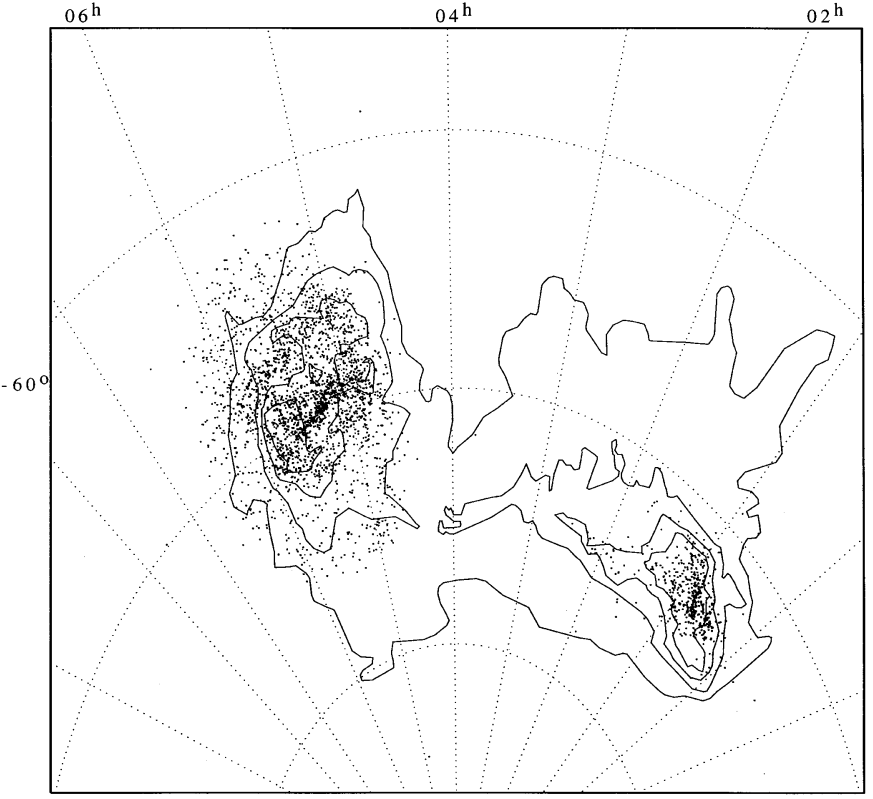

FIG. $4 b$

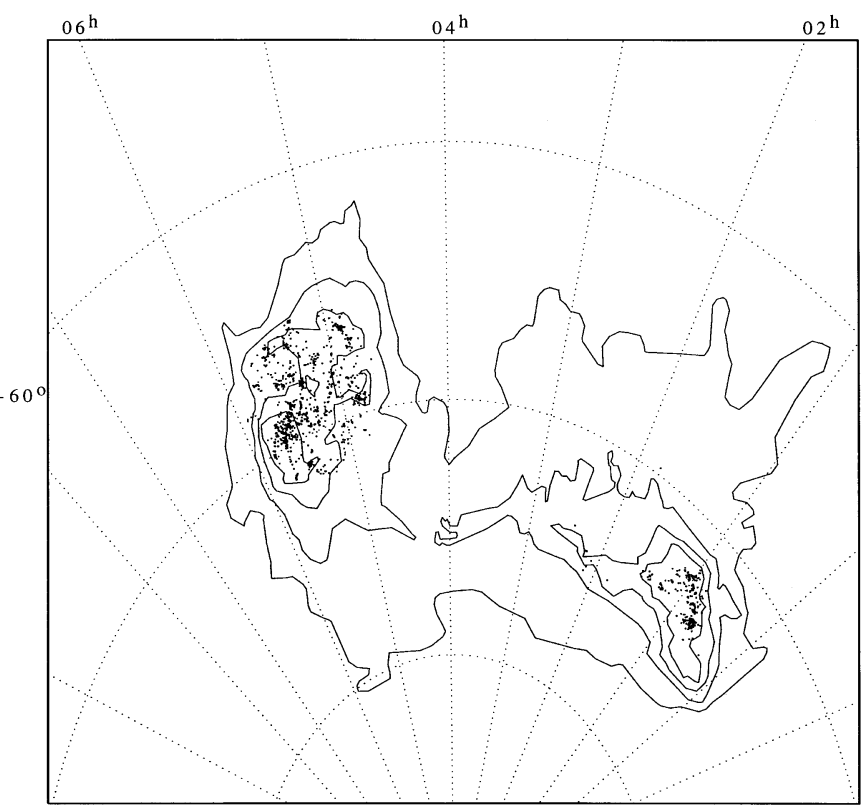

FIG. $4 d$

FIG. 4.-Magellanic System angular distributions of: $(a)$ all objects; $(b)$ star clusters; $(c)$ emission-free associations; $(d)$ objects with emission. H I surface density contours from Mathewson \& Ford (1984) as in Fig. 2. The declination tick marks are separated by $10^{\circ}$.

tions among previous catalogs, homogeneous measurements of positions, sizes and classification, which is important for future studies of their properties and for the identification of fainter and/or smaller objects. The angular distribution of object types is studied together with the $\mathrm{H} \mathrm{I}$ distribution. Asymmetries are present in the LMC disk, which consists of two off-centered systems with different inclinations, suggesting a warping. The previously identified structure in the LMC southern edge appears to be statistically significant. The present LMC catalog, together with that of the SMC and Bridge regions (Paper I), provide a total of 7847 objects, homogeneously cataloged. The analysis of the angular distribution of this ensemble provides interesting hints about the interaction between the Clouds.

We thank an anonymous referee for interesting remarks. We thank Miriani Pastoriza and Thaisa Storchi-Bergmann for the efforts to acquire the ESO/SERC R and J Atlases to the Instituto de Física, and Horacio Dottori for the magnifying lens that we used in the measurements. 
Battinelli, P., \& Demers, S. 1992, AJ, 104, 1458

Bhatia, R. K., \& Hatzidimitriou, D. 1988, MNRAS, 230, 215

Bhatia, R. K., \& MacGillivray, H. T. 1989, A\&A, 211, 9

Bhatia, R. K., Read, M. A., Hatzidimitriou, D., \& Tritton, S. 1991, A\&AS, 87,335

Bica, E., Clariá, J. J., \& Dottori, H. 1992, AJ, 103, 1859

Bica, E., Clariá, J. J., Dottori, H., Santos, J. F. C., Jr., \& Piatti, A. E. 1996, ApJS, 102, 57

Bica, E., Geisler, D., Dottori, H., Clariá, J. J., Piatti, A. E., \& Santos, J. F. C., Jr. 1998, AJ, 116, 723

Bica, E., \& Schmitt, H. R. 1995, ApJS, 101, 41 (Paper I)

Da Costa, G. S. 1991, in The Magellanic Clouds, ed. R. Haynes \& D. Milne (Dordrecht: Kluwer), 145

Davies, R. D., Elliot, K. H., \& Meaburn, J. 1976, MmRAS, 81, 89

de Vaucouleurs, G. 1955, AJ, 60, 126

Dottori, H. A., Bica, E., Clariá, J. J., \& Puerari, I. 1996, ApJ, 461, 742

Geisler, D., Bica, E., Dottori, H. A., Clariá, J. J., Piatti, A. E., \& Santos, J. F. C. Jr. 1997, AJ, 114, 1920

Grondin, L. Demers, S., \& Kunkel, W. E. 1992, AJ, 103, 1234

Hardy, E., Buonanno, R., Corsi, C. E., Janes, K. A., \& Schommer, R. A. 1984, ApJ, 278, 592

Henize, K. G. 1956, ApJS, 2, 315

Hernquist, L., \& Weil, M. L. 1993, MNRAS, 261, 804

Heydari-Malayeri, M., Grebel, E. K., Melnick, J., \& Jorda, L. 1993, A\&A, 278,11

Heydari-Malayeri, M., \& Hutsemékers, D. 1991, A\&A, 244, 64

Heydari-Malayeri, M., Magain, P., \& Remy, M. 1988, A\&A 201, L41 1989, A\&A 222, 41

Heydari-Malayeri, M., Niemela, V. S., \& Testor, G. 1987, A\&A, 184, 300

Heydari-Malayeri, M., \& Testor, G. 1983, A\&A, 118, 116

Hodge, P. W. 1960, ApJ, 131, 351

. 1973, AJ, 78, 807

1975, Irish AJ, 12, 77

. 1980, AJ, 85, 423 .1988, PASP, 100, 1051

Hodge, P. W., \& Sexton, J. A. 1966, AJ, 71, 363

Hodge, P. W., \& Wright, F. W. 1967, The Large Magellanic Cloud (Washington: Smithsonian Press)

Irwin, M. J. 1991, in IAU Symp. 148, The Magellanic Clouds, ed. R. Haynes \& D. Milne (Dordrecht: Kluwer), 453

Irwin, M. J., Demers, S., \& Kunkel, W. E. 1990, AJ, 99, 191

\section{REFERENCES}

Jenkner, H., Lasker, B. M., Struch, C. R., McLean, B. J., Shara, M. M., \& Russell, J. L. 1990, AJ, 99, 2081

Kontizas, M., Kontizas, E., Dapergolas, A., Argyropoulos, S., \& BellasVelidis, Y. 1994, A\&AS, 107, 77

Kontizas, E., Metaxa, M., \& Kontizas, M. 1988, AJ, 96, 1625

Kontizas, M., Morgan, D. H., Hatzidimitriou, D., \& Kontizas, E. 1990, A\&AS, 84, 527

Lauberts, A. 1982, The ESO/Uppsala Survey of the ESO(B) Atlas (Garching: ESO)

Lucke, P. B., \& Hodge, P. W. 1970, AJ, 75, 171

Lortet, M. C., \& Testor, G. 1984, A\&A, 139, 330

Lyngå, G., \& Westerlund, B. 1963, MNRAS, 127, 31

Mathewson, D. S., \& Ford, V. L. 1984, IAU Symp. 108, 125

Mathewson, D. S., Ford, V. L., Dopita, M. A., Tuohy, I. R., Long, K. S., \& Helfand, D. J. 1983, ApJS, 51, 345

Mathewson, D. S., Ford, V. L., Dopita, M. A., Tuohy, I. R., Mills, B. Y., \& Turtle, A. J. 1984, ApJS, 55, 189

Mathewson, D. S., Ford, V. L., Tuohy, I. R., Mills, B. Y., Turtle, A. J., \& Helfald, D. J. 1985, ApJS, 58, 197

Meaburn, J. 1980, MNRAS, 192, 365

Melnick, J. 1987, in IAU Symp. 121, Observational Evidence of Activity in Galaxies, ed. E. Ye. Khachikian, K. J. Fricke, \& J. Melnick (Dordrecht: Reidel), 1545

Oliveira, M. R., Dottori, H., \& Bica, E. 1998, MNRAS, 295, 921

Olszewski, E. W., Harris, H. C., Schommer, R. A., \& Canterna, R. W. 1988, AJ, 95,84

Quinn, P. J., Hernquist, L., \& Fullagar, D. P. 1993, ApJ, 403, 74

Robertson, J. W. 1974, A\&AS, 15, 261

Sanduleak, N. 1969, CTIO Obs Contrib. 89

Santiago, B. X., Elson, R. A. W., Sigurdsson, S., \& Gilmore, G. F. 1998, MNRAS, 295, 860

Shapley, H., \& Lindsay, E. M. 1963, Irish AJ, 6, 74

Smith, R. C., Chu, Y. H., Low, M.-M. M., Oey, M. S., \& Klein, U. 1994, AJ, 108,1266

van den Bergh, S. 1981, A\&AS, 46, 79

Westerlund, B. E. 1990, A\&AR, 2, 29

Wielen, R. 1971, A\&A, 13, 309

. 1988, in Globular Clusters in Galaxies, ed. J. E. Grindlay \& A. G. Davis Philip (Dordrecht: Reidel), 393

Zaritsky, D., Harris, J., \& Thompson, I. 1997, AJ, 114, 1002 